\title{
Paranasal Sinus Invasion in Nasopharyngeal Carcinoma after Intensity-Modulated Radiotherapy
}

\section{Caineng Cao, MD \\ Feng Jiang, $M D$ \\ Oifeng Jin, MD \\ Ting Jin, MD \\ Shuang Huang, MD \\ Qiaoying Hu, MD \\ Yuanyuan Chen, MD \\ Yongfeng Piao, MD \\ Yonghong Hua, MD \\ Xinglai Feng, MD \\ Xiaozhong Chen, MD}

Department of Radiation Oncology, Key Laboratory of Head \& Neck Cancer

Translational Research of Zhejiang Province,

Zhejiang Cancer Hospital, Hangzhou, China
Correspondence: Xiaozhong Chen, MD Department of Radiation Oncology, Key Laboratory of Head \& Neck Cancer Translational Research of Zhejiang Province, Zhejiang Cancer Hospital, No. 38, Guangji Road, Hangzhou 310022, China Tel: 86-571-88128202

Fax: 86-571-88122062

E-mail: chenxiaozhong2016@163.com

Received December 24, 2017

Accepted February 22, 2018

Published Online February 26, 2018

\section{Purpose}

The aim of this study is to evaluate the prognostic significance of paranasal sinus invasion for nasopharyngeal carcinoma (NPC) and its suitable position in the T classification.

\section{Materials and Methods}

The magnetic resonance imaging (MRI) scans of 695 patients with previously untreated, biopsy-proven, non-metastatic NPC that was treated with intensity-modulated radiotherapy (IMRT) were reviewed retrospectively.

\section{Results}

The incidence of paranasal sinus invasion was 39.4\% (274 of 695 patients). Multivariate analysis showed that paranasal sinus invasion was an independent negative prognostic factor for local failure-free survival (LFFS) $(p<0.05)$. According to the eighth American Joint Committee on Cancer (AJCC) staging system, 275 patients were classified as T3 classification. Of these, 78 patients (28.4\%) developed paranasal sinus invasion (T3b) and 197 (71.6\%) didn't (T3a). The estimated 5-year LFFS and overall survival (OS) rates for the patients with T3b and T3a classification were $88.6 \%$ versus $95.0 \%$ ( $p=0.047)$, and $84.5 \%$ versus $93.3 \%$ ( $p=0.183$ ), respectively. The estimated 5 -year LFFS and OS rates for the patients with T4 classification were $89.5 \%$ and $83.2 \%$, which were similar with the outcomes of patients with $\mathrm{T} 3 \mathrm{~b}$ classification.

\section{Conclusion}

MRI-determined paranasal sinus invasion is an independent prognostic factor of NPC treated by IMRT. Paranasal sinus invasion is recommended to classify as T4 classification in the 8th AJCC staging system for NPC.

\section{Introduction}

In China, nasopharyngeal carcinoma (NPC) is endemic and over 33,000 new patients were diagnosed in 2012, which accounted for $40 \%$ of new diagnosed NPC worldwide [1]. According to the eighth edition of the American Joint Committee on Cancer (AJCC) staging system published in 2017, $\mathrm{T}$ classification for NPC is based on the anatomical extent of the primary tumor and which has been proposed on NPC

\section{Key words}

Nasopharyngeal carcinoma, Paranasal sinuses, Intensity-modulated radiotherapy, Prognostic value, American Joint Committee on Cancer staging system

treated by intensity-modulated radiotherapy (IMRT) [2,3]. In the eighth edition of the AJCC staging system for NPC, paranasal sinus involvement is classified as $\mathrm{T} 3$ disease [2]. However, the Chinese 2008 staging system which has been proposed in the era of magnetic resonance imaging (MRI) and modern radiation therapy technology classifies involvement of the paranasal sinuses as T4 disease [4].

T4 classification NPC patients with MRI-detected intracranial extension are more likely to experience local failure and death after IMRT than patients without intracranial exten- 
Table 1. Patient characteristics

\begin{tabular}{|c|c|c|c|c|}
\hline Characteristic & $\begin{array}{l}\text { Whole group } \\
\qquad(n=695)\end{array}$ & $\begin{array}{l}\text { Non-PSI group } \\
\qquad(\mathrm{n}=421)\end{array}$ & $\begin{array}{l}\text { PSI group } \\
(n=274)\end{array}$ & p-value \\
\hline \multicolumn{5}{|l|}{ Sex } \\
\hline Male & $477(68.6)$ & $274(65.1)$ & $203(74.1)$ & 0.012 \\
\hline Female & $218(31.4)$ & $147(34.9)$ & $71(25.9)$ & \\
\hline \multicolumn{5}{|l|}{ Age (yr) } \\
\hline$<48$ & $329(47.3)$ & 209 (49.6) & $120(43.8)$ & 0.131 \\
\hline$\geq 48$ & $366(52.7)$ & $212(50.4)$ & $154(56.2)$ & \\
\hline \multicolumn{5}{|c|}{ Pathology classification } \\
\hline Keratinizing & $6(0.9)$ & $3(0.7)$ & $3(1.1)$ & 0.685 \\
\hline Non-keratinizing & $689(99.1)$ & 418 (99.3) & $271(98.9)$ & \\
\hline \multicolumn{5}{|l|}{ T classification } \\
\hline $\mathrm{T} 1$ & $107(15.4)$ & $107(25.4)$ & - & $<0.001$ \\
\hline $\mathrm{T} 2$ & $101(14.5)$ & $101(24.0)$ & - & \\
\hline T3 & $275(39.6)$ & $197(46.8)$ & $78(28.5)$ & \\
\hline $\mathrm{T} 4$ & $212(30.5)$ & $16(3.8)$ & $196(71.5)$ & \\
\hline \multicolumn{5}{|l|}{$\mathrm{N}$ classification } \\
\hline N0 & 79 (11.4) & $48(11.4)$ & $31(11.3)$ & 0.277 \\
\hline N1 & $304(43.7)$ & $174(41.3)$ & $130(47.4)$ & \\
\hline N2 & $212(30.5)$ & $131(31.1)$ & $81(29.6)$ & \\
\hline N3 & 100 (14.4) & $68(16.2)$ & $32(11.7)$ & \\
\hline \multicolumn{5}{|l|}{ Overall stage } \\
\hline I & $14(2.0)$ & $14(3.3)$ & - & $<0.001$ \\
\hline II & $94(13.5)$ & $94(22.3)$ & - & \\
\hline III & $299(43.0)$ & $231(54.9)$ & $68(24.8)$ & \\
\hline IVA & $288(41.4)$ & 82 (19.5) & $206(75.2)$ & \\
\hline
\end{tabular}

Values are presented as number (\%). PSI, paranasal sinus invasion.

sion in our previous study [5]. With respect to the prognostic value of paranasal sinus invasion, there are limited reports, especially, for patients treated by IMRT [6-10]. Therefore, we analyzed a large cohort of MRI-staged patients treated with IMRT to evaluate the prognostic significance of paranasal sinus invasion and its suitable position in the T classification.

\section{Materials and Methods}

\section{Patients and work-up}

After obtaining approval from our institutional review board, the medical records of consecutive 695 patients with previously untreated, biopsy-proven, non-metastatic NPC that was treated with IMRT between January 2007 and February 2012 in our center were retrospectively evaluated. All patients were restaged according to the eighth edition of the AJCC staging system. The pretreatment workup included a complete history and physical examination, hematology, and biochemistry profiles, MRI of the head and neck, computed tomography (CT) scan of the chest and abdominal region, bone scintigraphy, fiber-optic nasopharyngoscopy, and dental check. The clinical characteristics are listed in Table 1.

\section{MR imaging}

All patients underwent MRI on a 1.5- or 3.0-T system system (Magnetom Symphony/Verio, Siemens Healthcare, Erlangen, Germany) with a head-and-neck combined coil. The scan range covered from the suprasellar cistern to the inferior margin of the sternoclavicular joint. The thickness / slice gap was $5 \mathrm{~mm} / 1 \mathrm{~mm}$ for axial plane and $5 \mathrm{~mm} / 0.5 \mathrm{~mm}$ for sagittal and coronal plane, respectively. Non-enhanced series included: axial T1-weighted imaging (T1WI; fast spin echo [FSE], repetition time [TR]/echo time $[\mathrm{TE}]=460$ $\mathrm{msec} / 8.3 \mathrm{msec}$ ), sagittal T1WI (FSE, TR/TE=500 msec/9.0 $\mathrm{msec}$ ), axial T2-weighted imaging (T2WI; FSE with fat-suppressed, TR/TE=6,200 $\mathrm{msec} / 92.9 \mathrm{msec}$ ). After injection of gadopentetate dimeglumine with a dose of $0.2 \mathrm{~mL} / \mathrm{kg}$ and 
rate of $2.0 \mathrm{~mL} / \mathrm{sec}$, contrast-enhanced scan was performed by FSE with fat-suppressed (TR/TE $=460 \mathrm{msec} / 7.8 \mathrm{msec}$ for sagittal and coronal plane, TR/TE=295 msec/2.9 msec for axial plane). Two radiologists independently evaluated all scans, and any disagreements were resolved by consensus. In addition, there is a multidisciplinary team of NPC to confirm the extent of diseases and the treatment in our center.

Paranasal sinuses involvement was diagnosed using the following criteria: (1) primary tumors invaded into the sinus cavity with bone destruction of the wall of the sinus (Fig. 1) and (2) the same signal intensity characteristics as the primary lesion (an equal or lower signal in the T1WI MRI scan, an equal or higher signal in the T2WI and an obvious enhancement in the enhanced scan) [9].

\section{Treatment}

All patients received definitive radiotherapy using IMRT techniques. A detailed description of IMRT has been previously reported [11]. Briefly, the dose prescribed was 69-70.4 Gy, 63-67.2 Gy, 60-60.8 Gy, and 54-54.4 Gy in 30-32 fractions delivered over 6 weeks at the periphery of the planning target volume (PTV) of primary tumor, PTV of metastatic lymph nodes, PTV of high-risk clinical target volume, and PTV of low-risk clinical target volume, respectively, using the simultaneous integrated boost technique. Most patients $(\mathrm{n}=673,96.8 \%)$ underwent platinum-based neoadjuvant, concurrent, or adjuvant chemotherapy.

\section{Follow-up and statistical analysis}

Follow-up was calculated from the day of radiation therapy completion to the date of the event or the last follow-up visit. All patients were followed up after the completion of radiotherapy: 1 month after the completion of radiotherapy, every 3 months in the first 2 years, every 6 months from year 3 to year 5 , and annually thereafter.

The SPSS ver. 17.0 (SPSS Inc., Chicago, IL), software was used for statistical analysis. The local failure-free survival (LFFS), regional failure-free survival (RFFS), distant metastasis-free survival (DMFS), and overall survival (OS) were estimated by use of the Kaplan-Meier method. LFFS, RFFS, DMFS, and OS were measured from day 1 of treatment to the date of the event. Multivariate analysis was performed by using the Cox proportional hazards model. All statistical tests were two sided, and $\mathrm{p}<0.05$ was considered to be statistically significant.

\section{Ethical statement}

This study was approved by the Institutional Review Board of Zhejiang Cancer Hospital with a waiver of informed

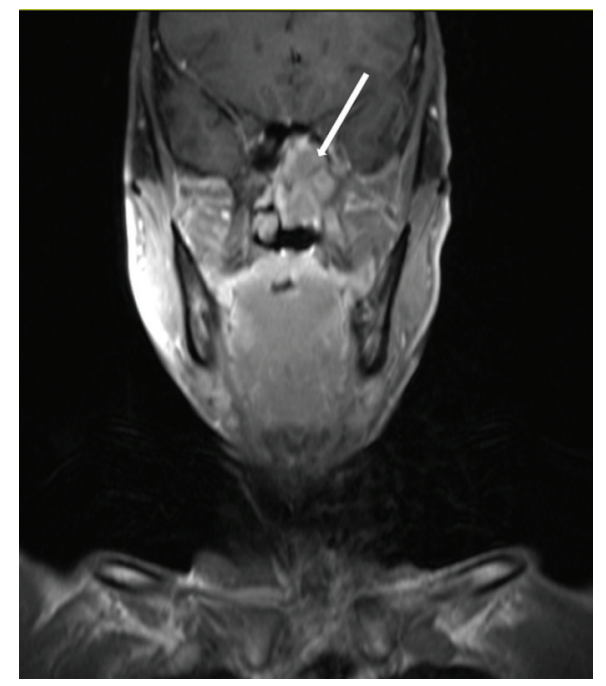

Fig. 1. Paranasal sinus invasion in one patient with nasopharyngeal carcinoma. A coronal contrast-enhanced T1-weighted magnetic resonance image in a 52-year-old man show the sphenoid sinus invasion (arrow).

consent (IRB No. IRB-2018-27) and performed in accordance with the principles of the Declaration of Helsinki.

\section{Results}

\section{Incidence of paranasal sinus invasion}

The incidence of paranasal sinus invasion was $39.4 \%$ (274 of 695 patients), with invasion of the ethmoid sinus, maxillary sinus and sphenoid sinus in $21(3.0 \%), 33(4.7 \%)$, and 272 $(39.1 \%)$ patients, respectively. None of patients had frontal sinus involvement. Of the 272 patients with sphenoid sinus invasion, $230(84.6 \%)$ did not have maxillary sinus or ethmoid sinus invasion. In contrast, of the 21 patients with ethmoid sinus invasion and 33 patients with maxillary sinus invasion, 20 (95.2\%) and $32(96.7 \%)$ also had sphenoid sinus invasion, respectively.

\section{Prognosis of patients with paranasal sinus invasion}

The median follow-up period was 63 months (range, 2 to 119 months). The estimated 5-year LFFS, RFFS, DMFS, and OS rates for the whole group were $92.0 \%, 93.5 \%, 87.5 \%$, and $89.1 \%$, respectively. The estimated 5-year LFFS, RFFS, DMFS, and OS rates for the patients without and with paranasal 
A

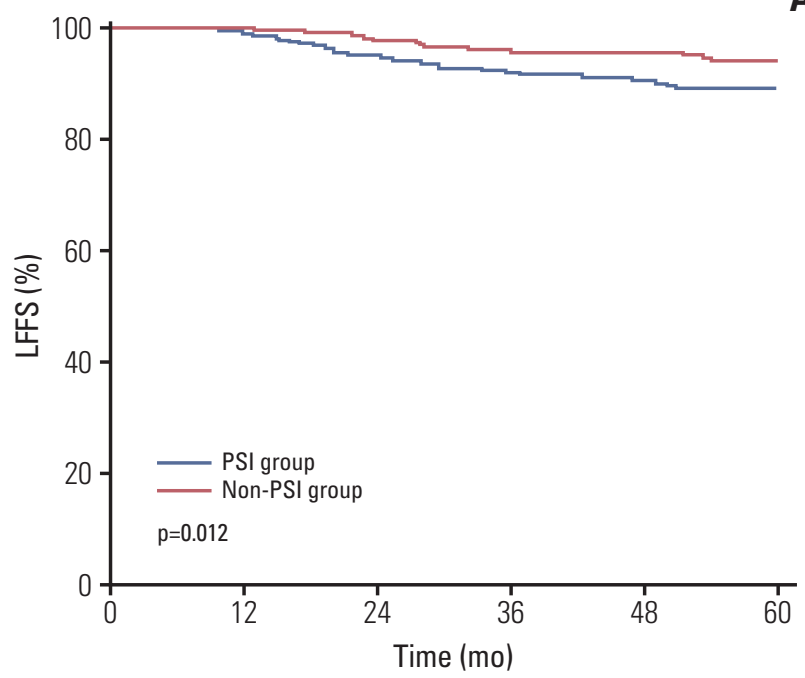

C

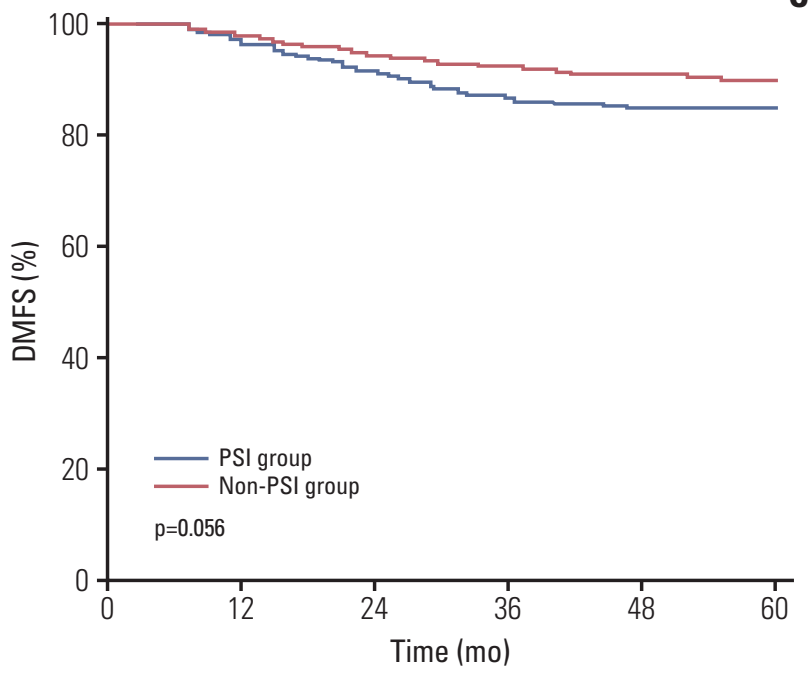

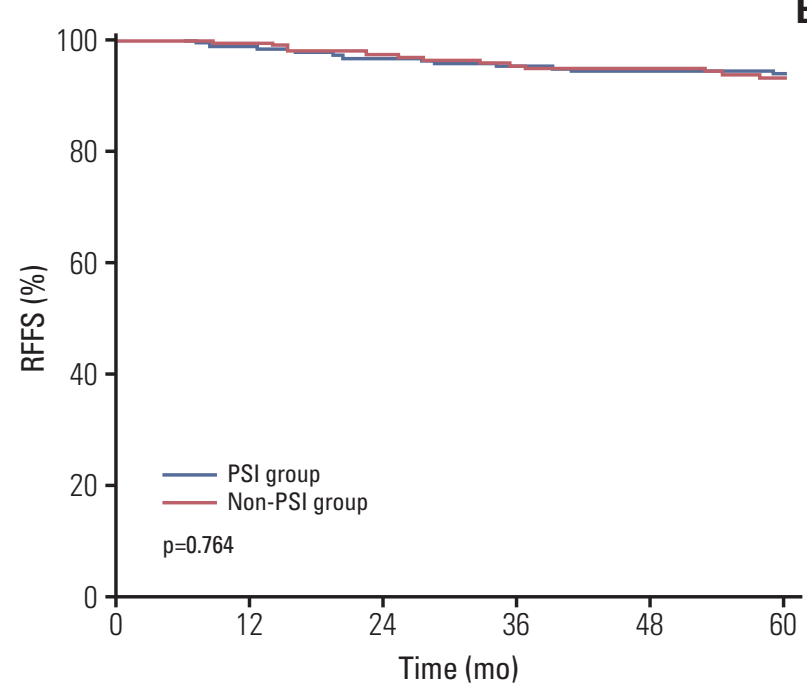

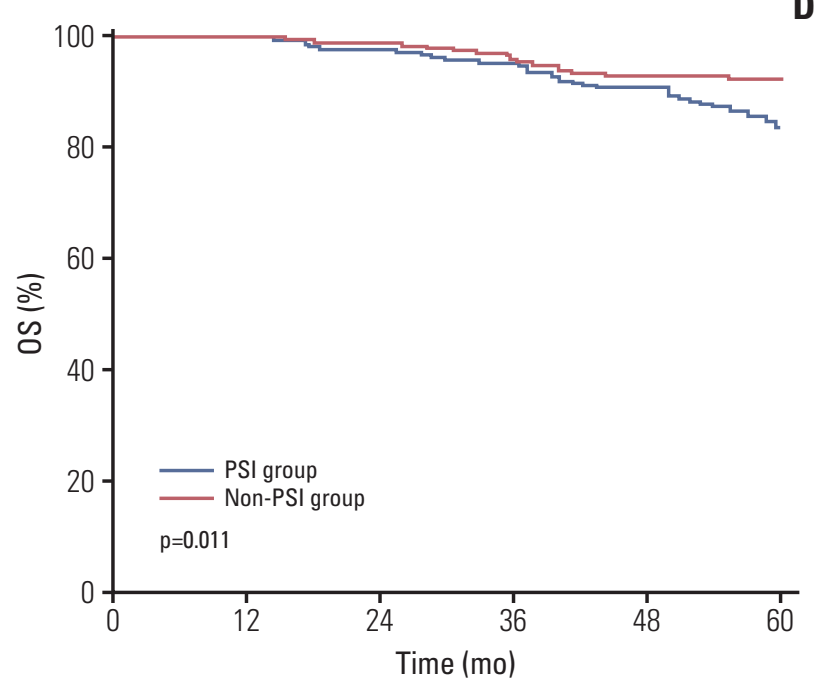

Fig. 2. Kaplan-Meier curve showing local failure-free survival (LFFS) (A), regional failure-free survival (RFFS) (B), distant metastasis-free survival (DMFS) (C), and overall survival (OS) rates (D) for the patients without and with paranasal sinus invasion (PSI) in the study.

sinus invasion were $93.9 \%$ versus $89.0 \%(\mathrm{p}=0.012), 93.2 \%$ versus $94.0 \%(\mathrm{p}=0.764), 89.3 \%$ versus $84.7 \%(\mathrm{p}=0.056)$, and $92.2 \%$ versus $83.7 \%$ ( $\mathrm{p}=0.011$ ), respectively (Fig. 2).

The value of various potential prognostic factors including age ( $\geq 48$ years and $<48$ years), sex, prevertebral muscles extension, medial pterygoid muscle extension, lateral pterygoid muscle extension, parapharyngeal space extension, skull base erosion, paranasal sinus invasion, involvement beyond the lateral surface of lateral pterygoid muscle, cranial nerve palsy, intracranial extension, $\mathrm{N}$ classification and con- current chemotherapy on predicting LFFS, RFFS, DMFS, and OS were evaluated. By multivariate analysis, paranasal sinus invasion was an independent negative prognostic factor for LFFS (Table 2).

\section{T classification of paranasal sinus invasion}

According to the eighth AJCC staging system, 275 patients were classified as T3 classification. Of these, 78 patients (28.4\%) developed paranasal sinus invasion (T3b) and 197 
Table 2. Multivariate analysis of variables correlated with various clinical endpoints

\begin{tabular}{llccr} 
Endpoint & \multicolumn{1}{c}{ Item } & HR & 95\% CI & p-value \\
LFFS & Paranasal sinus invasion & 1.945 & $1.104-3.426$ & 0.021 \\
RFFS & N classification & 1.536 & $1.116-2.115$ & 0.008 \\
DMFS & N classification & 1.681 & $1.311-2.156$ & $<0.001$ \\
& Cranial nerve palsy & 2.074 & $1.121-3.837$ & 0.020 \\
OS & Age $(\geq 48$ yr vs. $<48$ yr $)$ & 2.147 & $1.291-3.572$ & 0.003 \\
& Intracranial extension & 1.942 & $1.040-3.628$ & 0.037 \\
\hline
\end{tabular}

p-values were calculated by using the Cox proportional hazards model. HR, hazard ratio; CI, confidence interval; LFFS, local failure-free survival; RFFS, regional failure-free survival; DMFS, distant metastasis-free survival; OS, overall survival.

A
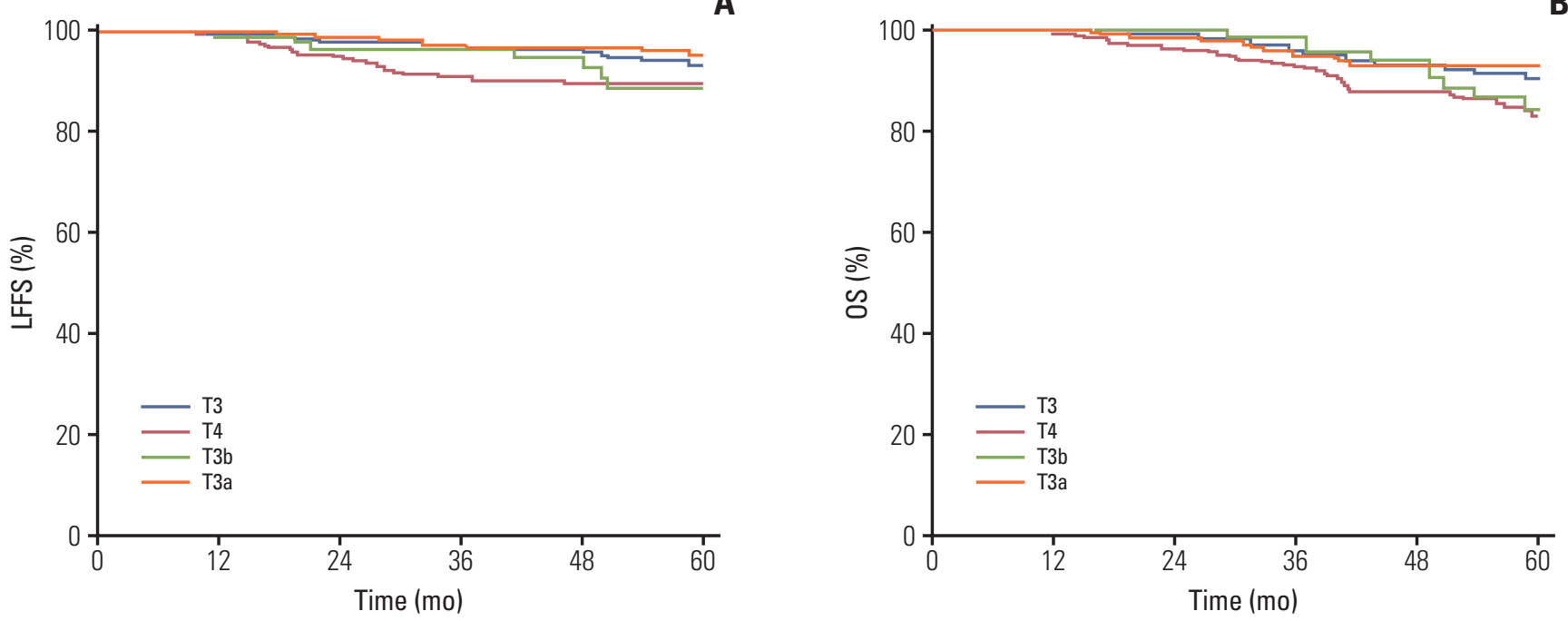

Fig. 3. Probability of local failure-free survival (LFFS) (A) and overall survival (OS) rates (B) for patients with T3 classification and T4 classification according to the eighth American Joint Committee on Cancer (AJCC) staging system. T3b, T3 patients with paranasal sinus invasion; T3a, T3 patients without paranasal sinus invasion.

$(71.6 \%)$ didn't (T3a). The estimated 5-year LFFS and OS rates for the patients with T3b and T3a classification were $88.6 \%$ versus $95.0 \%$ ( $\mathrm{p}=0.047$ ), and $84.5 \%$ versus $93.3 \%$ ( $\mathrm{p}=0.183$ ), respectively. The estimated 5-year LFFS and OS rates for the patients with $\mathrm{T} 4$ classification were $89.5 \%$ and $83.2 \%$, which were similar with the outcomes of patients with T3b classification (Fig. 3).

Of the 274 patients with paranasal sinus invasion, most of patients with maxillary sinus or ethmoid sinus invasion simultaneously accompanied with sphenoid sinus invasion. Patients with paranasal sinus invasion were classified as patients with invasion of the sphenoid sinus alone, without invasion of the maxillary sinus or ethmoid sinus (group A, $\mathrm{n}=230$ ), and patients with invasion of the maxillary sinus and / or ethmoid sinus (group B, $\mathrm{n}=44$ ). The $\mathrm{T}$ and $\mathrm{N}$ classifi- cation distribution according to paranasal sinus invasion is shown in Table 3. No significant differences were observed in LFFS, RFFS, DMFS, and OS between the groups A and B (5-year LFFS, $88.6 \%$ vs. 90.7\%, $\mathrm{p}=0.778$; 5-year RFFS, 93.3\% vs. $97.1 \%, \mathrm{p}=0.393$; 5-year DMFS, $85.0 \%$ vs. $83.0 \%$, $\mathrm{p}=0.896$; 5-year OS, $82.1 \%$ vs. $91.5 \%$, $\mathrm{p}=0.130$ ).

\section{Discussion}

In this study, we observed a high incidence of paranasal sinus invasion in NPC and that paranasal sinus invasion is an independent prognostic factor of LFFS in NPC after IMRT. 
Table 3. Distribution of $\mathrm{T}$ and $\mathrm{N}$ classifications with paranasal sinus invasion

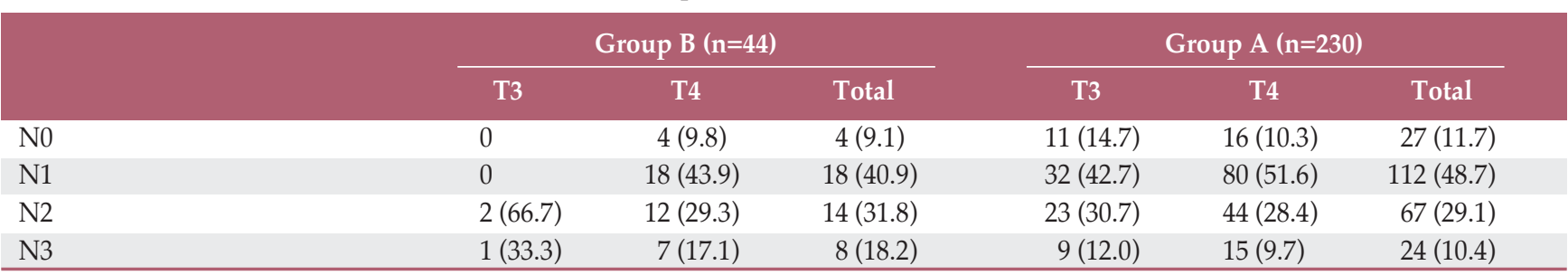

Values are presented as number $(\%)$.

In patients with $\mathrm{T} 3$ classification, tumors with paranasal sinus invasion had a higher risk of local failure than those without paranasal sinus invasion. Paranasal sinus invasion was recommended to classify as T4 classification in the eighth AJCC staging system for NPC.

The result of the present study indicated that the incidence of paranasal sinus invasion in NPC was $39.4 \%$. The highest rate of incidence was of sphenoid sinus invasion $(39.1 \%)$, followed by maxillary sinus invasion $(4.7 \%)$ and ethmoid sinus invasion (3.0\%). Compared to the respective rates of $15 \%$ $27 \%, 4.9 \%-9 \%$, and $4 \%-14 \%$ reported in previous studies [6-10], the rate of sphenoid sinus invasion was higher in the current study. The floor of the sphenoid sinus borders the nasopharynx roof. No muscle or fascia to act as a barrier against tumor invasion, tumors originating from the nasopharynx tend to spread directly and superiorly into the sphenoid sinus. This accounts for the high rate of incidence of sphenoid sinus invasion. In the diagnosis of gross paranasal sinus invasion, there should be minimal discrepancy. However, the diagnosis of subtle paranasal sinus invasion may pose a considerable diagnostic challenge and a source of variation between different radiologists or centers. Compared with CT, MRI has been used to assess the extent of NPC more reliably and accurately [12-14]. All of the patients in the present study were evaluated by MRI, which was recommended as the preferred modalities for staging [15]. Variations in the diagnosis of paranasal sinus invasion may explain this inconsistency in the different studies.

In the Chinese 1992 staging system for NPC, patients with paranasal sinus invasion were defined as $\mathrm{T} 4$, and this classification remains in the Chinese 2008 staging system. The present study indicated that the estimated 5-year LFFS and OS rates for the patients with $\mathrm{T} 4$ classification were similar with the outcomes of patients with T3b classification. Paranasal sinus invasion was recommended to classify as T4 classification in the eighth AJCC staging system. In a study of 1,811 patients with NPC treated by IMRT, tumors with ethmoid or maxillary sinus invasion which were recommended to classify as T4 classification had a higher risk of local failure than those with sphenoid sinus invasion alone [10]. Only three patients with maxillary sinus and/or ethmoid sinus invasion were classified as T3 classification (Table 3). For NPC after IMRT, locoregional recurrence rates at 1, 2, 3, 4, and 5 years accounted for $14.7 \%, 49.4 \%, 66.7 \%, 85.4 \%$, and $94.7 \%$ of the total, respectively [16]. The 3-year local relapsefree survival rate was reported in the previous study [10]. It is reasonable to report the 5-year survival outcomes for prognostic analysis. No significant differences were observed in LFFS, RFFS, DMFS, and OS between the patients with invasion of the sphenoid sinus alone, without invasion of the maxillary sinus or ethmoid sinus and patients with invasion of the maxillary sinus and/or ethmoid sinus, which was probably associated with the aid of MRI, IMRT, and the use of chemotherapy [17-19].

There are several limitations in the current study, including the retrospective nature of the study design and the inclusion of patients treated at a single center. In China, only $2 \%$ patients had keratinizing disease, which accounts for up to $67 \%$ of NPC in western countries [20]. The effect of paranasal sinus invasion on the prognosis and staging of patients with NPC should be further confirmed by other cohorts from different regions.

MRI-determined paranasal sinus invasion is an independent prognostic factor of NPC treated by IMRT. Paranasal sinus invasion is recommended to classify as T4 classification in the 8th AJCC staging system for NPC.

\section{Conflicts of Interest}

Conflict of interest relevant to this article was not reported.

\section{Acknowledgments}

The present study was supported by Zhejiang Province Medical and Health Science and Technology Project (2017182785). 


\section{References}

1. International Agency for Research on Cancer. GLOBOCAN 2012: estimated cancer incidence, mortality and prevalence worldwide in 2012. Lyon: IARC Press; 2012.

2. Amin MB, Edge S, Greene F, Byrd DR, Brookland RK, Washington MK, et al. AJCC cancer staging manual. 8th ed. New York: Springer; 2017.

3. Pan JJ, Ng WT, Zong JF, Chan LL, O'Sullivan B, Lin SJ, et al. Proposal for the 8th edition of the AJCC/UICC staging system for nasopharyngeal cancer in the era of intensity-modulated radiotherapy. Cancer. 2016;122:546-58.

4. Chinese Committee for Staging of Nasopharyngeal Carcinoma. Report on revision of the Chinese 1992 staging system for nasopharyngeal carcinoma. J Radiat Oncol. 2013;2:233-40.

5. Cao C, Luo J, Gao L, Yi J, Huang X, Li S, et al. Magnetic resonance imaging-detected itracranial extension in the T4 classification nasopharyngeal carcinoma with intensity-modulated radiotherapy. Cancer Res Treat. 2017;49:518-25.

6. Chong VF, Fan YF, Khoo JB. Computed tomographic and magnetic resonance imaging findings in paranasal sinus involvement in nasopharyngeal carcinoma. Ann Acad Med Singapore. 1998;27:800-4.

7. King AD, Lam WW, Leung SF, Chan YL, Teo P, Metreweli C. MRI of local disease in nasopharyngeal carcinoma: tumour extent vs tumour stage. Br J Radiol. 1999;72:734-41.

8. Tao CJ, Liu X, Tang LL, Mao YP, Chen L, Li WF, et al. Prognostic scoring system for locoregional control among the patients with nasopharyngeal carcinoma treated by intensitymodulated radiotherapy. Chin J Cancer. 2013;32:494-501.

9. Tian L, Li YZ, Mo YX, Liu LZ, Xie CM, Liang XX, et al. Nasopharyngeal carcinoma with paranasal sinus invasion: the prognostic significance and the evidence-based study basis of its T-staging category according to the AJCC staging system. BMC Cancer. 2014;14:832.

10. Zhang Y, Peng H, Guo R, Li WF, Chen L, Liu X, et al. Should all nasopharyngeal carcinoma with paranasal sinus invasion be staged as T3 in the intensity-modulated radiotherapy era? A study of 1811 cases. J Cancer. 2016;7:1353-9.
11. Jin T, Zhang Q, Jiang F, Qin WF, Jin QF, Cao CN, et al. Neoadjuvant chemotherapy with different dose regimens of docetaxel, cisplatin and fluorouracil (TPF) for locoregionally advanced nasopharyngeal carcinoma: a retrospective study. Oncotarget. 2017;8:100764-72.

12. Chung NN, Ting LL, Hsu WC, Lui LT, Wang PM. Impact of magnetic resonance imaging versus $\mathrm{CT}$ on nasopharyngeal carcinoma: primary tumor target delineation for radiotherapy. Head Neck. 2004;26:241-6.

13. Abdel Khalek Abdel Razek A, King A. MRI and CT of nasopharyngeal carcinoma. AJR Am J Roentgenol. 2012;198: 11-8.

14. Lee AW, Sze WM, Au JS, Leung SF, Leung TW, Chua DT, et al. Treatment results for nasopharyngeal carcinoma in the modern era: the Hong Kong experience. Int J Radiat Oncol Biol Phys. 2005;61:1107-16.

15. Chua ML, Wee JT, Hui EP, Chan AT. Nasopharyngeal carcinoma. Lancet. 2016;387:1012-24.

16. Sun X, Su S, Chen C, Han F, Zhao C, Xiao W, et al. Long-term outcomes of intensity-modulated radiotherapy for 868 patients with nasopharyngeal carcinoma: an analysis of survival and treatment toxicities. Radiother Oncol. 2014;110: 398-403.

17. Chen L, Liu LZ, Mao YP, Tang LL, Sun Y, Chen Y, et al. Grading of MRI-detected skull-base invasion in nasopharyngeal carcinoma and its prognostic value. Head Neck. 2011;33: 1309-14.

18. Cao CN, Luo JW, Gao L, Yi JL, Huang XD, Wang K, et al. Update report of $\mathrm{T} 4$ classification nasopharyngeal carcinoma after intensity-modulated radiotherapy: an analysis of survival and treatment toxicities. Oral Oncol. 2015;51:190-4.

19. Lee AW, Ma BB, Ng WT, Chan AT. Management of nasopharyngeal carcinoma: current practice and future perspective. J Clin Oncol. 2015;33:3356-64.

20. Shedd DP, Von Essen CF, Eisenberg H. Cancer of the nasopharynx in Connecticut, 1935 through 1959. Cancer. 1967;20: 508-11. 